\title{
Environment with reduced ion bombardment energy for levitated particles in an rf plasma
}

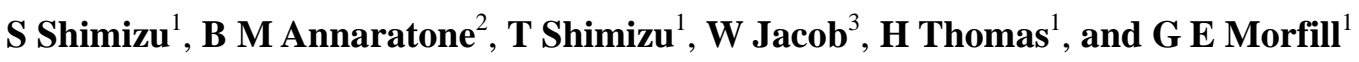 \\ ${ }^{1}$ Max-Planck-Institut für extraterrestrische Physik, Gießenbachstraße, D-85748 Garching, \\ Germany \\ ${ }^{2}$ Laboratoire de Physique des Interactions Ioniques et Moléculaires, CNRS/Université de \\ Provence, Centre de St. Jêrome case 321, 13397, Marseille, France \\ ${ }^{3}$ Max-Planck-Institut für Plasmaphysik, Boltzmannstraße 2, D-85748 Garching, Germany \\ E-mail: sshimizu@mpe.mpg.de
}

\begin{abstract}
We describe levitation of diamond fine particles in the $\mathrm{H}_{2} \mathrm{rf}$ plasma chamber equipped with an adaptive rf electrode. Since suppression of ion bombardment is essential for crystalline diamond growth, we use an adaptive rf electrode system in a parallel-plate capacitively coupled rf plasma in order to levitate particles in a quasineutral "spot plasma" region. Here the ions energy corresponds only to the floating potential of the particles without the additional energy of the streaming ions as in the sheath. One can expect ion bombardment with greatly reduced ion energy when this technique is applied to diamond deposition on levitated particles.
\end{abstract}

PACS: 52.27.Lw, 52.80.Pi

Published in: $\quad$ Plasma Sources Science and Technology 17, 035014 (4pp) (2008)

Received: $\quad 2$ October 2007, in final form 31 March 2008

Published: $\quad 26$ June 2008

Online at $\quad$ stacks.iop.org/PSST/17/035014

Doi: $\quad$ 10.1088/0963-0252/17/3/035014 


\section{INTRODUCTION}

Fine particles with diameters of a few micrometers in a plasma are negatively charged and sensitive to the balance of several forces, e.g., gravity, electrostatic, thermophoretic, ion drag and others $[1,2]$. Therefore, choosing proper plasma parameters allows levitation of particles in the electric field of the plasma sheath. The precise experimental parameters for levitation depend strongly on the particles' size and shape [3] as well as on the type of gas and the plasma parameters. For example, it is possible to form Coulomb crystals particles cloud at proper conditions [4].

Nishimura et al, observed successful levitation of fine diamond particles in a hydrogen plasma [5], although normally their non-spherical shape makes levitation difficult due to anisotropic forces [3]. Hayashi et al, observed the growth of carbon materials on the levitated amorphous carbon particles in an rf plasma [6]. Those results lead to the possibility of homoepitaxial growth of diamonds on particles levitated in a $\mathrm{CH}_{4} / \mathrm{H}_{2}$ plasma. This deposition, not on a planar substrate (two-dimensional, 2D) but on levitated particles (3D), is technologically interesting. Kersten et al reported the aluminum particles growth using the "3D" method [7].

In a previous study we reported growth of diamond grains on levitated seed particles, using an rf plasma chemical vapor deposition (CVD) with $\mathrm{CH}_{4}$ and $\mathrm{H}_{2}$ [8]. To enhance the quality of CVD diamond, the energy of the ions arriving at the growth surface should be as low as possible. For example, it was shown that the diamond nucleation density is small in an environment with high energy ion bombardment [9]. In another study, we reported the manipulation of levitated particles by an adaptive if or dc electrode [10, 11]. Particles are levitated in a "spot plasma region" where they are surrounded by a glow that indicates the presence of electrons. Ionization happens around the seeds and cold ions are created at a sheath distance from the surface. Even if they are created a bit further, the charge exchange cross section for cold ions is much greater than for accelerated ions. That means they do not acquire extra energy from the field. Therefore, in this experiment, ions hit the particle only with the energy of the particle sheath without the additional energy of the streaming ions (as in the usual ground based experiment) and one can expect ion bombardment with significantly reduced ion energy. Such an environment appears to be suitable for "3D" diamond growth. In general, CVD diamond is grown with the gas ratio of $\mathrm{CH}_{4} / \mathrm{H}_{2}=1 \%$, i.e. hydrogen plasma dominant condition. In this article, we demonstrate levitation of diamond fine particles in the rf $\mathrm{H}_{2}$ plasma chamber equipped with an adaptive rf electrode. 


\section{EXPERIMENTAL}

The levitation of diamond fine particles was carried out in a parallel-plate capacitively coupled rf hydrogen plasma chamber equipped with an adaptive if electrode. Figure 1 shows it's schematic set-up. The top electrode is driven by rf voltage at $13.56 \mathrm{MHz}$ and the bottom electrode is grounded. At the center of the bottom electrode, an adaptive electrode is mounted, to which rf voltage, of variable amplitude and phase with respect to the upper electrode rf, is applied. $\mathrm{H}_{2}$ gas is supplied through a mass flow controller into the chamber. After the plasma is ignited between the top and bottom electrodes, diamond fine particles (Nilako, \#4000, $\phi=2-4 \mu \mathrm{m}$ (non-spherical)) are supplied from the particles dispenser. Then, an rf voltage is applied to the adaptive electrode to make a spot plasma. A He-Ne laser illuminates the system for visualizing the levitated particles. The particles’ position and motion are monitored by a CCD camera which detects the laser light scattered from the levitated particles.

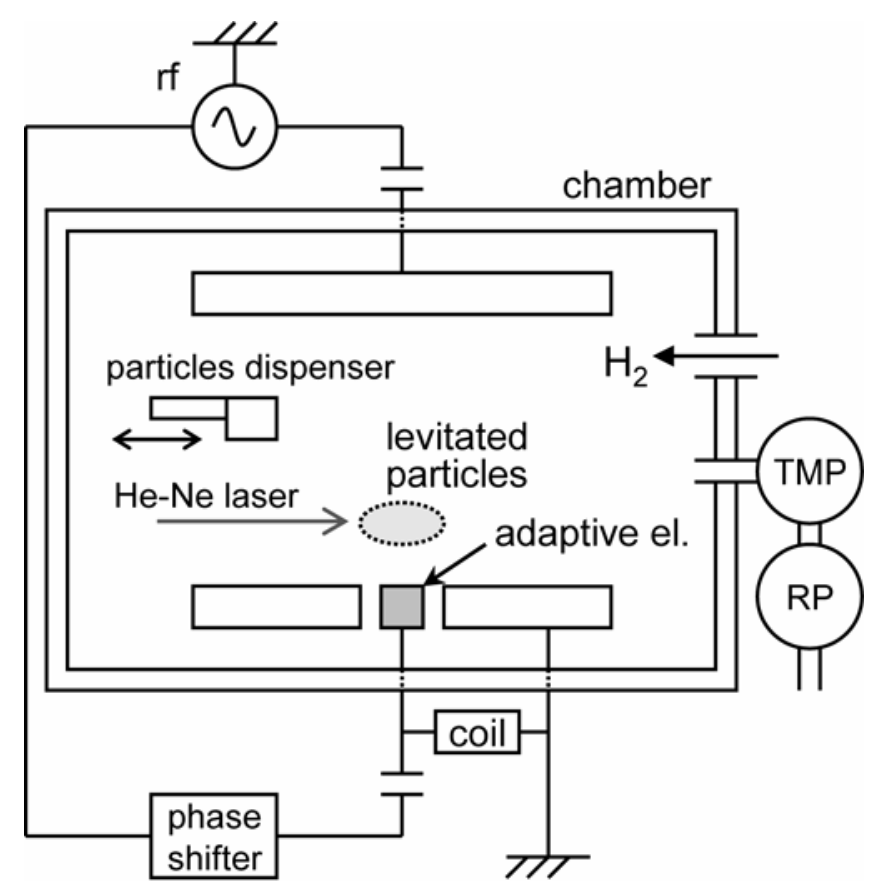

Figure 1. Schematic set-up of the rf plasma chamber equipped with an adaptive rf electrode. 


\section{RESULTS AND DISCUSSION}

\subsection{Levitation of diamond fine particles in hydrogen rf plasmas with an adaptive rf electrode}

The levitation of diamond fine particles was investigated in the rf plasma chamber equipped with an adaptive rf electrode. CVD diamond growth is generally performed using $\mathrm{CH}_{4}(1 \%)$ and $\mathrm{H}_{2}$ (99 \%) gas mixture, therefore, a $\mathrm{H}_{2}$ plasma was chosen for our experiment. The results are shown in Figures 2 (a) - (c). The levitation conditions are the following: (a) without a spot plasma (only a main rf plasma is ignited between the bottom and top electrodes) (b) main plasma + spot plasma (voltage on the adaptive electrode: $182 \mathrm{~V}$, phase shift with respect to the top electrode: $17^{\circ}$ ) (c) main plasma + spot plasma (voltage: $206 \mathrm{~V}$, phase shift: $25^{\circ}$ ). The other conditions are: $\mathrm{H}_{2}=1$ sccm, pressure: $100 \mathrm{~Pa}$, rf voltage applied on the bottom electrode $=290 \mathrm{~V}$. The bottom and top electrodes are at room temperature. Without spot plasma, particles are levitated at the main plasma sheath (Fig. 2(a)). With the weekly ignited spot plasma, particles are trapped partially there (Fig. 2(b)). With the strongly ignited spot plasma, all the particles are trapped there (Fig. 2(c)).

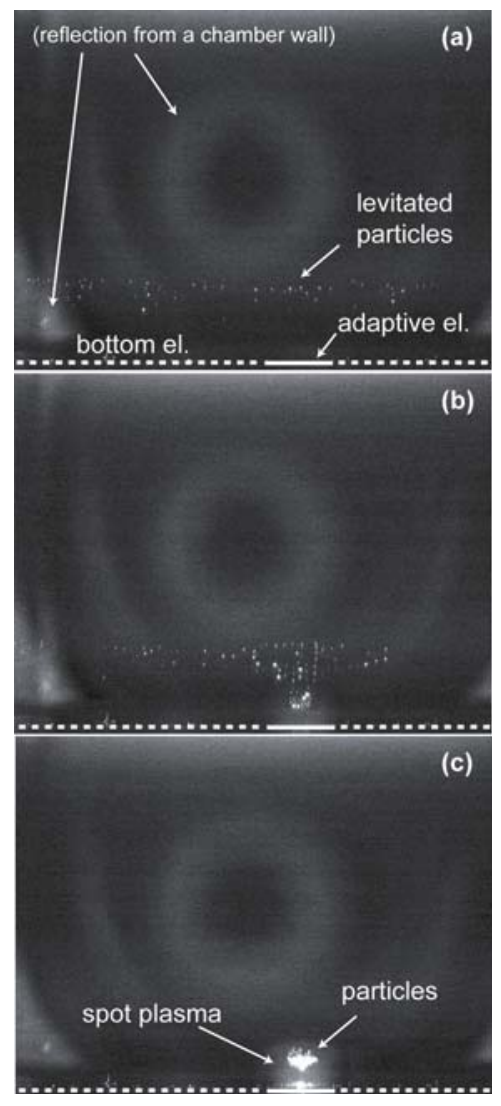

Figure 2. Pictures of the levitated diamond fine particles in the rf hydrogen plasma equipped with an adaptive rf electrode. The surfaces of the adaptive and bottom electrodes are indicated by solid and dashed lines, respectively. Particles are levitated under the following conditions: (a) without a spot plasma (only a main rf plasma is ignited between the bottom and top electrodes) (b) main plasma + spot plasma (voltage on the adaptive electrode: $182 \mathrm{~V}$, phase shift with respect to the top electrode: $17^{\circ}$ ) (c) main plasma + spot plasma (voltage: 206 V, phase shift: $25^{\circ}$ ). 


\subsection{Estimation of ion bombardment energy}

Here we analyse theoretically the energy of the ions impinging on the particles in three distinct cases: 1) particles laying on the substrate, 2) particles levitated in the plasma sheath, and 3) particles levitated in the spot plasma. In case (1) the substrate is negative with respect to the plasma with a voltage which is, at least, equal to the floating potential for plane geometry. This value can be greatly increased by radio frequency self bias. The ions pick up energy from the field in a mean free path $\left(\lambda_{\mathrm{mfp}}\right)$ that can be calculated from the energy dependent collision cross section. At the total pressure of $100 \mathrm{~Pa}$, the value of these experiments, a rough estimate of the collisionality can be derived using Cramer's total cross sections [12]. For ions accelerated to $5 \mathrm{eV}$ the mean free path is about $0.05 \mathrm{~mm}$ which gives 4 collisions in a $0.2 \mathrm{~mm}$ sheath. The energy acquired by an ion between two collisions is the potential difference across the sheath, about $20 \mathrm{eV}$, divided by the number of the collisions. The result, $5 \mathrm{eV}$, gives an intrinsic self consistency to the estimate.

In case (2) the particles settle in plasma sheath and at the edge of the light emission region. Here the potential is the plasma potential reduced by an average excitation energy. This has to be the case, because at this energy the number of the electrons able to make excited species and hence cause light emission decreases exponentially. Levitation of particles is obtained when the balance of all forces acting on the particles are in equilibrium. This means that the sum of all other forces equal gravity. Among the other forces such as electrostatic force, ion drag, thermophoresis, etc., the electrostatic forces $\left(F_{\mathrm{e}}\right)$ are usually dominant. $F_{\mathrm{e}}$ is given by

$$
F_{e}=Q E,
$$

where $Q$ is the equilibrium charge of the solid particle induced by the plasma and $E$ is the ambient electrostatic field. For $\lambda_{\mathrm{D}} / \lambda_{\mathrm{mfp}}=1 \sim 2$, as in our case, moderately collision region is applicable ( $\lambda_{\mathrm{D}}$ : Debye length). The edge of the visible emission region corresponds to the edge of the quasineutral pre-sheath region from which ions exit with the Bohm speed, $\left(k T_{\mathrm{e}} / M\right)^{1 / 2}\left(T_{\mathrm{e}}\right.$ : electron temperature, $M$ : ion mass) acquired in the last mean free path [13]. At this point, the field increases monotonically up to the transition region field.

$$
E=\frac{k T_{e} / e}{\lambda_{m f p}^{3 / 5} \lambda_{D}^{2 / 5}}
$$


with $k T_{\mathrm{e}} / e=3 \mathrm{~V}, \lambda_{\mathrm{mfp}}=10^{-4} \mathrm{~m}$ and $\lambda_{\mathrm{D}}=10^{-4} \mathrm{~m}$, this field is $\sim 10^{4} \mathrm{~V} / \mathrm{m}$ and the ions acquire an energy of the order of $1 \mathrm{eV}$. This energy is higher than the energy acquired in the sheath of the particle, see case (3). The energy reported in Table 1 for case (2) are hence underestimated of a quantity of the order of case (3). The detailed results are summarized and compared in Table 1.

In case (3) the particles are levitating in the plasma, in our case in the spot plasma that forms above the adaptive electrode driven by rf. Ions are produced locally by the intense ionisation and because of the high charge exchange cross section, slow ions are accelerated only in the sheath that forms around the particles [14]. In Argon at $100 \mathrm{~Pa}$ the mean free path for ion-neutral collisions far from the probe (low $T_{\mathrm{i}}$ (ion temperature) $\sim 0.03 \mathrm{eV}$ ) is of the order of $0.015 \mathrm{~mm}$ (cross section 2.15 $\times 10^{-18} \mathrm{~m}^{2}$ ) [15]. The extension of the sheath is dependent on the model for the ion acceleration, a recent relevant publication is by [16], however, for a ratio $r_{\mathrm{p}} / \lambda_{\mathrm{D}} \sim 10^{-2}$ ( $r_{\mathrm{p}}$ : particle radius) typically the sheath develops in distances smaller than $\lambda_{\mathrm{D}}$, in our case $\sim 10^{-4} \mathrm{~m}$, see for example [17]. Therefore, the energy picked up by the ions is lower than the floating potential energy. Moreover in the spot plasma the grains are electrostatically strongly coupled (see fig. 2(c)), i.e. the inter-grain distances are less than $\lambda_{\mathrm{D}}$, as in our case, the charge of the grain and the floating potential are even further reduced. This leads to much lower ion impact energies.

The energies acquired by the ions in the three cases for hydrogen and argon are summarized in Table 1. In case (3), the floating potential is calculated using the radial motion theory [14], which looks preferable for our condition, or the collision less case of the orbital motion limited theory (OML) for comparison [18]. We can see that the third case results in the lowest ion energies. Therefore, using an rf adaptive electrode, one can expect to obtain a more "benign" environment during diamond growth on levitated particles with $\mathrm{CH}_{4} / \mathrm{H}_{2}$ plasmas. It should be added that the $\lambda_{\text {mfp }}$ of hydrogen $(E=0.03 \mathrm{eV})$ is calculated with the capture cross-section value at the ion energy of $0.1 \mathrm{eV}$ in reference [19], therefore, the $E_{\text {ion }}$ values of hydrogen in case (3) contain slight errors. On the other hand, simple extrapolation of the capture cross-section curve to a lower ion energy region gives smaller $\lambda$ and $E_{\text {ion }}$ than those shown in Table 1 . 
Table 1. Comparison of the energy acquired by the ions in the three cases for Hydrogen and Argon. Case (1): the average energy is calculated as $V_{\mathrm{f}-\mathrm{planar}} \times \lambda_{\mathrm{mfp}}(E=5 \mathrm{eV}) / L$, with $L$ the sheath thickness (estimated from our experiment, $0.2 \mathrm{~mm}$ ). Case (2): $E \times \lambda_{\mathrm{mfp}}\left(E=5 \mathrm{eV}\right.$ ) (see the main text for the calculation of $E$ ). Case (3): $V_{\mathrm{f}}$ $\times \lambda_{\text {mfp }}(E=0.03 \mathrm{eV}) / \lambda_{\mathrm{D}}$ where $V_{\mathrm{f}}$ (floating potential) is calculated using the orbital motion limited theory $\left(V_{\mathrm{f}-\mathrm{OML}}\right)$ or the radial motion theory $\left(V_{\mathrm{f} \text {-radial }}\right)$. Other parameters: $T_{\mathrm{e}}=3 \mathrm{eV}$, total pressure $=100 \mathrm{~Pa}$ and temperature $=300 \mathrm{~K}$.

\begin{tabular}{cccccccccc}
\hline gas & $\begin{array}{c}V_{\mathrm{f}-\mathrm{planar}} \\
\text { case }(1) \\
(\mathrm{ion})\end{array}$ & $\begin{array}{c}V_{\mathrm{f}-\mathrm{OML}} \\
\text { case }(3) \\
(\mathrm{V})\end{array}$ & $\begin{array}{c}V_{\text {f-radial }} \\
\text { case }(3) \\
(\mathrm{V})\end{array}$ & $\begin{array}{c}\lambda_{\mathrm{mfp}} \\
(E=5 \mathrm{eV}) \\
(\mu \mathrm{m})\end{array}$ & $\begin{array}{c}\lambda_{\mathrm{mfp}} \\
(E=0.03 \mathrm{eV}) \\
(\mu \mathrm{m})\end{array}$ & $\begin{array}{c}E_{\text {ions }} \\
\text { case }(1) \\
(\mathrm{eV})\end{array}$ & $\begin{array}{c}E_{\text {ions }} \\
\text { case }(2) \\
(\mathrm{eV})\end{array}$ & $\begin{array}{c}E_{\text {ions-OML }} \\
\text { case }(3) \\
(\mathrm{eV})\end{array}$ & $\begin{array}{c}E_{\text {ions-radial }} \\
\text { case (3) } \\
(\mathrm{eV})\end{array}$ \\
\hline $\begin{array}{c}\mathrm{H}_{2} \\
\left(\mathrm{H}^{+}\right)\end{array}$ & 10.0 & $3.8^{\mathrm{a}}$ & $0.3^{\mathrm{b}}$ & $111^{\mathrm{c}}$ & $\begin{array}{c}40^{\mathrm{c}} \\
(E=0.1 \mathrm{eV})\end{array}$ & 5.6 & 1.8 & 0.38 & 0.03 \\
$\begin{array}{c}\mathrm{Ar} \\
\left(\mathrm{Ar}^{+}\right)\end{array}$ & 15.5 & $7.3^{\mathrm{a}}$ & $1.5^{\mathrm{b}}$ & $50^{\mathrm{d}}$ & $19^{\mathrm{e}}$ & 3.9 & 1.3 & 0.35 & 0.07 \\
\hline
\end{tabular}

${ }^{\mathrm{a}}$ Reference 18

${ }^{\mathrm{b}}$ Reference 14

${ }^{\mathrm{c}}$ Reference 19

${ }^{\mathrm{d}}$ Reference 12

e Reference 15

\section{CONCLUSIONS}

We confirmed levitation of diamond fine particles in the rf hydrogen plasma chamber equipped with an adaptive if electrode. Since the electric field surrounding a particle in a spot plasma is less than that in typical plasma sheaths, one can expect ion bombardment with greatly reduced ion energy when this technique is applied to diamond deposition on levitated particles.

\section{ACKNOWLEDGEMENTS}

The authors would like to thank T. Antonova (Max-Planck-Institut fuer extraterrestrische Physik) for the experiment with the adaptive electrode system. 


\section{REFERENCES}

[1] Rothermel H, Hagl T, Morfill G E, Thoma M H and Thomas H M 2002 Phys. Rev. Lett. 89 175001

[2] Zafiu C, Melzer A and Piel A 2002 Phys. Plasmas 94794

[3] Annaratone B M and Morfill G E 2003 J. Phys. D: Appl. Phys. 362853

[4] Thomas H, Morfill G E, Demmel V and Goree J 1994 Phys. Rev. Lett. 73652

[5] Nishimura G, Iizuka S, Shimizu T and Sato N 2003 Diamond Relat. Mater. 12374

[6] Hayashi Y and Takahashi K 1997 Japan. J. Appl. Phys. 364976

[7] Kersten H, Wiese R, Thieme G, Fröhlich M, Kopitov A, Bojic D, Scholze F, Neumann H, Quaas M, Wulff H and Hippler R 2003 New J. Phys. 593

[8] Shimizu T, Jacob W, Thomas H, Morfill G, Abe T, Watanabe Y and Sato N 2006 Thin Solid Films 506-507 652

[9] Teii K and Yoshida T 1999 J. Appl. Phys 851864

[10] Annaratone B M, Anttonova T, Goldbeck D D, Thomas H M and Morfill G E 2004 Plasma Phys. Control. Fusion 46 B495

[11] Annaratone B M, Glier M, Stuffler T, Thomas H, Raif M and Morfill G E 2003 New J. Phys. 5 92.1

[12] Cramer H W 1959 J. Chem. Phys. 30641

[13] Riemann K U 1997 Phys. Plasmas 44158

[14] Kennedy R V and Allen J E 2002 J. Plasma Physics 67243

[15] Wobschall D, Graham jr. J R and Malone D P 1963 Phys. Rev. 1311565

[16] Arinaminpathy N, Allen J E and Ockendon J R 2007 J. Plasma Physics 73793

[17] Daugherty J E, Porteous R K, Kilgore M D and Gravesa D B 1992 J. Appl. Phys. 723934

[18] Allen J E 1992 Physical Scripta 45497

[19] Phelps A V 1990 J. Phys. Chem. Ref. Data 19653 